\title{
Erratum to: Fitting net photosynthetic light-response curves with Microsoft Excel - a critical look at the models
}

\author{
F. de A. LOBO ${ }^{*,+}$, M.P. de BARROS ${ }^{* *}$, H.J. DALMAGRO ${ }^{* *}$, Â.C. DALMOLIN**, W.E. PEREIRA ${ }^{* * *}$, \\ É.C. de SOUZA ${ }^{\#}$, G.L. VOURLITIS ${ }^{\# \#, ~ a n d ~ C . E . ~ R O D R I ́ G U E Z ~ O R T I ́ Z ~}{ }^{\# \#}$ \\ Departamento de Solos e Engenharia Rural, FAMEV/UFMT, 78060-900, Cuiabá-MT, Brasil* \\ Programa de Pós-Graduação em Física Ambiental, IF/UFMT, 78060-900, Cuiabá-MT, Brasil ${ }^{* *}$ \\ Departamento de Ciências Fundamentais e Sociais, CCA/UFPB, 58397-000, Areia-PB, Brasil*** \\ Departamento de Estatística, ICET/UFMT, 78060-900, Cuiabá-MT, Brasil ${ }^{\#}$ \\ Department of Biological Science, CSUSM, San Marcos-CA, 92096-0001, USA \#\# \\ Departamento de Botânica e Ecologia, IB/UFMT, 78060-900, Cuiabá-MT, Brasil ${ }^{\# \#}$
}

\section{Erratum to: Photosynthetica DOI: 10.1007/s11099-013-0045-y}

A few errors in Table 3, equations 14,16, 18, 25, 29, and 38, and two supplementary files (ESM4 and ESM9) were found in the above-mentioned paper. The corrected table and equations are published here. However, these errors do not compromise the discussion and analysis in the paper.

The publisher and authors apologize for these errors and for inconveniences they may have caused.

Table 3. The variables calculated from the models. $I_{\text {comp }}-$ light compensation point $\left[\mu\right.$ mol(photon) $\left.\mathrm{m}^{-2} \mathrm{~s}^{-1}\right] ; I_{\max }-$ light saturation point beyond which there is no significant change in $P_{\mathrm{N}}\left[\mu \mathrm{mol}\left(\right.\right.$ photon) $\left.\mathrm{m}^{-2} \mathrm{~s}^{-1}\right] ; I_{\text {sat }}-$ light saturation point $\left[\mu \operatorname{mol}\left(\right.\right.$ photon) $\left.\mathrm{m}^{-2} \mathrm{~s}^{-1}\right] ; I_{\text {sat }(50)}-$ light saturation point for $A+R_{\mathrm{D}}$ equal to $50 \%$ of $P_{\mathrm{N} \max }$ [ $\mu$ mol(photon) $\left.\mathrm{m}^{-2} \mathrm{~s}^{-1}\right] ; I_{\mathrm{sat}(85)}-$ light saturation point for $A+R_{\mathrm{n}}$ equal to $85 \%$ of $P_{\mathrm{N} m a x}$ [ $\mu$ mol(photon) $\mathrm{m}^{-2} \mathrm{~s}^{-1}$ ]; $I_{\mathrm{sat}(90)}-$ light saturation point for $A+R_{\mathrm{n}}$ equal to $90 \%$ of $P_{\mathrm{N} \text { max }}\left[\mu\right.$ mol(photon) $\left.\mathrm{m}^{-2} \mathrm{~s}^{-1}\right]$; $I_{\mathrm{sat}(95)}$ light saturation point for $A+R_{\mathrm{D}}$ equal to $95 \%$ of $P_{\mathrm{N} \max }\left[\mu \operatorname{mol}\left(\right.\right.$ photon) $\mathrm{m}^{-2} \mathrm{~s}^{-1}$; $P_{\mathrm{gmax}}-$ maximum gross photosynthetic rate $\left[\mu \mathrm{mol}\left(\mathrm{CO}_{2}\right) \mathrm{m}^{-2} \mathrm{~s}^{-1}\right] ; P_{\mathrm{N}(I \max )}-$ maximum net photosynthetic rate obtained at $I=I_{\max }\left[\mu \mathrm{mol}\left(\mathrm{CO}_{2}\right) \mathrm{m}^{-2} \mathrm{~s}^{-1}\right] ; R_{\mathrm{D}}-$ dark respiration $\left[\mu \mathrm{mol}\left(\mathrm{CO}_{2}\right) \mathrm{m}^{-2} \mathrm{~s}^{-1}\right] ; \phi_{\left(I_{\text {comp }}\right)}-$ quantum yield at $I=I_{\text {comp }}\left[\mu \mathrm{mol}\left(\mathrm{CO}_{2}\right) \mu \mathrm{mol}(\text { photon })^{-1}\right] ; \phi\left(I_{\text {comp }}-I_{200}\right)$ - quantum yield at the range between $I_{\text {comp }}$ and $I=200 \mu \mathrm{mol}(\mathrm{photon}) \mathrm{m}^{-2} \mathrm{~s}^{-1}$ $\left[\mu \operatorname{mol}\left(\mathrm{CO}_{2}\right) \mu \operatorname{mol}(\text { photon })^{-1}\right] ; \phi\left(I_{0}\right)$ - quantum yield at $I=0 \mu \operatorname{mol}\left(\right.$ photon) $\left.\mathrm{m}^{-2} \mathrm{~s}^{-1}\left[\mu \operatorname{mol}\left(\mathrm{CO}_{2}\right) \mu \text { mol(photon }\right)^{-1}\right]$; $\phi\left(I_{0}-I_{\text {comp }}\right)-$ quantum yield at the range between $I=0 \mu$ mol(photon) $\mathrm{m}^{-2} \mathrm{~s}^{-1}$ and $I_{\text {comp }}\left[\mu \mathrm{mol}\left(\mathrm{CO}_{2}\right) \mu \mathrm{mol}(\text { photon })^{-1}\right]$. *Photosynthetic active radiation values above the range employed to make the measurements. ${ }^{* *}$ Photosynthetic active radiation values above the maximum that reaches the Earth's surface.

\begin{tabular}{|c|c|c|c|c|c|c|c|c|c|}
\hline \multicolumn{10}{|c|}{ Calculated variabMathematical models } \\
\hline & Eq. 1 & Eq. 2 & Eq. 3 & Eq. 4 & Eq. 5 & Eq. 6 & Eq. 8 & Eq. 9 & Eq. 11 \\
\hline$I_{\text {comp }}$ & 22.5 & 22.5 & 22.7 & 20.2 & 20.2 & 23.4 & 22.0 & & \\
\hline$I_{\text {sat }(50)}$ & 261.4 & 261.4 & 209.4 & 211.0 & 211.0 & 235.6 & 210.1 & 210.1 & 211.1 \\
\hline$I_{\text {sat }(85)}$ & $1,376.3$ & $1,376.3$ & 559.2 & 462.4 & 462.4 & $1,022.7$ & 537.0 & 537.0 & 678.0 \\
\hline$I_{\mathrm{sat}(90)}$ & $2,172.6^{*}$ & $2,172.6^{*}$ & 713.1 & 539.8 & 539.8 & $1,564.3$ & 647.0 & 647.0 & 854.6 \\
\hline$I_{\mathrm{sat}(95)}$ & $4,561.7^{* *}$ & $4,561.7^{* *}$ & $1,047.6$ & 668.7 & 668.7 & $3,179.1^{* *}$ & 835.2 & 835.2 & $1,148.4$ \\
\hline$I_{\text {sat }}$ & & & & & & & & & $2,289.9^{*}$ \\
\hline$I_{\max }$ & 1537.0 & $1,949.0$ & $1,030.0$ & 847.0 & 847.0 & $1,348.0$ & $1,008.0$ & $1,008.0$ & $1,297.0$ \\
\hline$P_{\operatorname{gmax}}$ & & & & & & & & & 17.1 \\
\hline$P_{\mathrm{N}(I \max )}$ & 15.2 & 15.7 & 14.5 & 14.4 & 14.4 & 14.9 & 14.5 & 14.5 & 14.9 \\
\hline$R_{\mathrm{D}}$ & & & & & & & & & 1.7 \\
\hline$\phi\left(I_{0}\right)$ & & 0.0899 & & & 0.0433 & & & 0.0597 & 0.0824 \\
\hline$\phi\left(I_{\text {comp }}\right)$ & 0.0738 & 0.0738 & 0.0490 & 0.0431 & 0.0431 & 0.0638 & 0.0550 & 0.0574 & 0.0694 \\
\hline$\phi\left(I_{0}-I_{\text {comp }}\right)$ & 0.0816 & 0.0816 & 0.0492 & 0.0432 & 0.0432 & 0.0674 & 0.0573 & 0.0586 & 0.0757 \\
\hline$\phi\left(I_{\text {comp }}-I_{200}\right)$ & 0.0410 & 0.0410 & 0.0416 & 0.0391 & 0.0391 & 0.0421 & 0.0400 & 0.0410 & 0.0409 \\
\hline
\end{tabular}

The online version of the original article can be found under doi: 10.1007/s11099-013-0045-y

The erratum includes two corrected supplementary files.

${ }^{+}$Corresponding author; e-mail: f_a lobo@ufmt.br 
$I_{\text {sat }}=\frac{\sqrt{\frac{(\beta+\gamma) \times\left(1+\gamma \times I_{\text {comp }}\right)}{\beta}}-1}{\gamma}$

$\phi_{(I)}=\frac{I_{(50)} \times P_{\operatorname{gmax}}}{\left(I+I_{(50)}\right)^{2}}$

$\phi_{(I)}=\phi_{\left(I_{0}\right)} \times \operatorname{sech}^{2}\left(\frac{\phi_{\left(I_{0}\right)} \times I}{P_{\operatorname{gmax}}}\right)=\phi_{\left(I_{0}\right)} \times\left[\frac{1}{\cosh ^{2}\left(\frac{\phi_{\left(I_{0}\right)} \times I}{P_{\operatorname{gmax}}}\right)}\right]$

$I_{\text {comp }}=\frac{I_{(50)} \times R_{\mathrm{D}}}{P_{\text {gmax }}-R_{\mathrm{D}}}$

$I_{\text {comp }}=\frac{R_{\mathrm{D}} \times\left(\theta \times R_{\mathrm{D}}-P_{\text {gmax }}\right)}{\phi_{\left(I_{0}\right)} \times\left(R_{\mathrm{D}}-P_{\text {gmax }}\right)}$

$I_{\text {sat }(n)}=I_{\text {comp }}-\frac{\ln \left\{1-\left[\frac{\frac{n}{100} \times\left(P_{\text {gmax }}-R_{\mathrm{D}}\right)+R_{\mathrm{D}}}{P_{\text {gmax }}}\right]\right\}}{k}$ 